\title{
Principios lógicos y principios morales: la identidad de los indiscernibles
}

\author{
RAÚL QUESADA \\ Facultad de Filosofía y Letras \\ Universidad Nacional Autónoma de México \\ rquesada@lycos.com
}

\begin{abstract}
Resumen: Partiendo de la definición de la identidad en Principia Mathematica, aquí se discute el estatus lógico del principio de la identidad de los indiscernibles con los criterios y argumentos de Leibniz y Clarke. En la defensa que hace Leibniz de este principio se establece la distinción entre una necesidad lógica y una necesidad moral, relacionada con la existencia y el buen juicio de Dios, que se resume en el principio de razón suficiente; esta posición se puede relacionar con el Tractatus, donde se critica a Russell en términos similares a los que Clarke usó en contra de Leibniz. Wittgenstein, sin embargo, habla de la experiencia necesaria para entender la lógica: la experiencia fundamental de que algo es; así, se puede sugerir un paralelo entre su posición y la distinción de Leibniz, el cual también nos llevaría a replantear y repensar en términos leibnizianos la independencia radical de la lógica.
\end{abstract}

Palabras clave: Dios, existencia, necesidad, ética

\begin{abstract}
The logical status of the identity of indiscernibles is considered in the context of Principia Mathematica's definition of identity and in reference to the Leibniz-Clarke polemics. In his defence Leibniz distinguishes between logical and moral necessity and relates the latter to existence, jure divino, and the principle of sufficient reason. In Wittgenstein's Tractatus Russell is criticised in the same terms that Clarke used against Leibniz, but Wittgenstein also talks about the 'experience' that something is, necessary to understand logic, and points out that logic could be prior to the question 'How?', but not to the question 'What?' From this a parallel is suggested between Wittgenstein's position and Leibniz's distinction which could be used to rethink in Leibnizian terms logic's radical independence.
\end{abstract}

Key words: God, necessity, existence, ethics

I have nothing to say and I am saying it

JOHN CAGE

En Principia Mathematica, Russell ofrece la siguiente definición de la identidad:

$$
x=y .=:(\phi): \phi ! x . \supset . \phi ! y \quad D f .
$$

En el contexto teórico en el que se formula, esta definición tiene dos dificultades, una ontológica y otra lógica, que se suelen advertir desde una primera lectura. La dificultad ontológica tiene que ver con el estatus de la variable $\phi$ : ¿servirá para hablar de predicados, de propiedades, de funciones o de atributos?; en todo caso, ¿cómo caracterizaríamos tales entidades? La dificultad lógica se relaciona, por un lado, con la posibilidad de que la cuantificación universal propicie la generación de paradojas, y, por otro, 
con los remedios russellianos para tal mal: la noción de función predicativa, la ramificación de la teoría simple de los tipos y el axioma de reducibilidad. En este trabajo trataremos de situar estas dificultades dentro de un contexto menos técnico y más tradicional que nos permita vislumbrar otro tipo de presupuestos teóricos y, en consecuencia, otros aspectos filosóficos del problema.

En términos generales podríamos decir que la definición de Russell caracteriza la identidad a través de dos implicaciones: la primera dice que si $x$ y $y$ son idénticas, entonces no hay ninguna propiedad que no compartan, esto es, son indiscernibles; mientras que la segunda afirma que si $x$ y y son indiscernibles, entonces son idénticas. Así, la definición se podría descomponer en dos partes, la primera afirmaría que:

$$
x=y \rightarrow(\phi)(\phi ! x \supset \phi ! y)
$$

y la segunda que:

$$
(\phi)(\phi ! x \supset \phi ! y) \rightarrow x=y
$$

La primera de estas implicaciones se suele aceptar como esencial para cualquier caracterización de la identidad, aun cuando, como ya señalamos, se pueda objetar el carácter irrestricto o restringido de la cuantificación y los compromisos ontológicos de la cuantificación de segundo orden. La segunda implicación, por su parte, no sólo ha producido reparos, sino que más de una vez ha sido rechazada por considerarla simplemente falsa o por no ser necesaria. Pero tal vez su falsedad no sea tan simple, ya que, también más de una vez, ha logrado colarse en una definición lógica, y, falsa, verdadera o necesaria, gracias a Leibniz ha adquirido el estatus de principio. ${ }^{1}$ Leibniz hizo esta afirmación, conocida como el principio de la identidad de los indiscernibles, de diferentes maneras, pero en todas ellas es clara su afinidad fundamental con la formulación contemporánea: ${ }^{2}$

$$
(\phi)(\phi A \equiv \phi B) \rightarrow A=B
$$

Algunas de las formulaciones de Leibniz son las siguientes:

"que no es cierto que dos substancias se parezcan enteramente y sean diferentes solo numero" (Discurso de Metafísica, 9), 1685-1686; ${ }^{3}$

"no hay en la Naturaleza dos Seres que sean perfectamente el uno como el otro, y donde no sea posible encontrar una diferencia interna

${ }^{1}$ Afirma Leibniz: "Esos dos grandes principios, el de razón suficiente y el de la identidad de los indiscernibles, cambian el estado de la metafísica. Esa ciencia se hace real y demostrativa por medio de estos principios, mientras que antes, en general, consistía en palabras vacías" (Leibniz, Escrito IV, 5, en Alexander (comp.), The Leibniz-Clarke Correspondence, p. 37). [Las traducciones de las citas de esta obra son mías.]

${ }^{2}$ Véase Hidé Ishiguro, Leibniz's Philosophy of Logic and Language, p. 17.

${ }^{3}$ Leibniz, Discurso de Metafísica, p. 34. 
o fundamentada en una denominación intrínseca" (Monadología, 9), $1714 ;^{4}$

"En las cosas absolutamente indiferentes no hay alternativa y, consecuentemente, no hay elección, ni voluntad, puesto que la alternativa debe estar fundada en alguna razón o principio" (Escrito IV, 1), $1716 ;^{5}$

"No existe algo así como dos individuos indiscernibles uno del otro" (Escrito IV, 4), 1716; 6

"Suponer dos cosas indiscernibles es suponer la misma cosa bajo dos nombres" (Escrito IV, 6), 1716.

Este principio, claro está, lo formula Leibniz dentro del contexto de una compleja posición filosófica en la que las nociones lógicas y metafísicas van de la mano y nos guían hacia una epistemología que debemos poner en relación con sus ascendientes lógico-matemáticos. ${ }^{8}$ En el caso del principio de la identidad de los indiscernibles (PII, de aquí en adelante), el mismo Leibniz no sólo señala su carácter paradójico, tanto en su enunciación como en su relación con otras tesis, sino que considera que el principio se deriva o depende de otras tesis o principios también bastante radicales y discutibles, como el principio de que cada mónada refleja todo el universo y el principio de razón suficiente. Así, por ejemplo, el primer fragmento citado se encuentra en el siguiente contexto:

"QUE CADA SUBSTANCIA SINGULAR EXPRESA TODO EL UNIVERSO A SU MANERA, Y QUE EN SU NOCIÓN TODOS SUS ACONTECIMIENTOS ESTÁN COMPRENDIDOS CON TODAS SUS CIRCUNSTANCIAS Y TODA LA SERIE DE LAS COSAS EXTERIORES." Se siguen de esto varias paradojas considerables; entre otras, que no es cierto que dos substancias se parezcan enteramente y sean diferentes solo numero y que lo que Santo Tomás asegura a este respecto de los ángeles o inteligencias (quod ibi omne individuum sit species infima), es verdadero de todas las substancias mientras se tome la diferencia específica como la toman los geómetras respecto a sus figuras. [...] Además, toda substancia es como un mundo completo y como un espejo de Dios; o bien, de todo el universo que cada una de ellas expresa a su manera, algo así como una misma ciudad es vista de diferente manera según las diversas situaciones del que la contempla. [...] Puede decirse, incluso, que toda substancia lleva en cierta manera el carácter de la sabiduría infinita y la omnipotencia de Dios y lo imita en cuanto es capaz. Pues expresa,

${ }^{4}$ Leibniz, Monadología, p. 28.

${ }^{5}$ Alexander (comp.), op. cit, p. 36.

${ }^{6}$ Ibid.

${ }^{7}$ Ibid., p. 37

${ }^{8}$ Véanse, por ejemplo, B. Russell, A Critical Exposition of the Philosophy of Leibniz; G.H.R. Parkinson, Logic and Reality in Leibniz's Metaphysics; H. Ishiguro, Leibniz's Philosophy of Logic and Language. 
con los remedios russellianos para tal mal: la noción de función predicativa, la ramificación de la teoría simple de los tipos y el axioma de reducibilidad. En este trabajo trataremos de situar estas dificultades dentro de un contexto menos técnico y más tradicional que nos permita vislumbrar otro tipo de presupuestos teóricos y, en consecuencia, otros aspectos filosóficos del problema.

En términos generales podríamos decir que la definición de Russell caracteriza la identidad a través de dos implicaciones: la primera dice que si $x$ y $y$ son idénticas, entonces no hay ninguna propiedad que no compartan, esto es, son indiscernibles; mientras que la segunda afirma que si $x$ y y son indiscernibles, entonces son idénticas. Así, la definición se podría descomponer en dos partes, la primera afirmaría que:

$$
x=y \rightarrow(\phi)(\phi ! x \supset \phi ! y)
$$

y la segunda que:

$$
(\phi)(\phi ! x \supset \phi ! y) \rightarrow x=y
$$

La primera de estas implicaciones se suele aceptar como esencial para cualquier caracterización de la identidad, aun cuando, como ya señalamos, se pueda objetar el carácter irrestricto o restringido de la cuantificación y los compromisos ontológicos de la cuantificación de segundo orden. La segunda implicación, por su parte, no sólo ha producido reparos, sino que más de una vez ha sido rechazada por considerarla simplemente falsa o por no ser necesaria. Pero tal vez su falsedad no sea tan simple, ya que, también más de una vez, ha logrado colarse en una definición lógica, y, falsa, verdadera o necesaria, gracias a Leibniz ha adquirido el estatus de principio. ${ }^{1}$ Leibniz hizo esta afirmación, conocida como el principio de la identidad de los indiscernibles, de diferentes maneras, pero en todas ellas es clara su afinidad fundamental con la formulación contemporánea: ${ }^{2}$

$$
(\phi)(\phi A \equiv \phi B) \rightarrow A=B
$$

Algunas de las formulaciones de Leibniz son las siguientes:

"que no es cierto que dos substancias se parezcan enteramente y sean diferentes solo numero" (Discurso de Metafísica, 9), 1685-1686; ${ }^{3}$

"no hay en la Naturaleza dos Seres que sean perfectamente el uno como el otro, y donde no sea posible encontrar una diferencia interna

${ }^{1}$ Afirma Leibniz: "Esos dos grandes principios, el de razón suficiente y el de la identidad de los indiscernibles, cambian el estado de la metafísica. Esa ciencia se hace real y demostrativa por medio de estos principios, mientras que antes, en general, consistía en palabras vacías" (Leibniz, Escrito IV, 5, en Alexander (comp.), The Leibniz-Clarke Correspondence, p. 37). [Las traducciones de las citas de esta obra son mías.]

${ }^{2}$ Véase Hidé Ishiguro, Leibniz's Philosophy of Logic and Language, p. 17.

${ }^{3}$ Leibniz, Discurso de Metafísica, p. 34. 
objeción tendremos que mencionar la segunda característica del PII que, dijimos, nos puede ayudar a entender mejor la perspectiva desde la que se formula; esta característica es su íntima relación con el Principio de Razón Suficiente (PRS): debe haber una razón suficiente por la cual todo es como es y no de otra manera. ${ }^{12}$

De este principio deriva Leibniz la afirmación de que no pueden existir dos seres que sean totalmente indiscernibles, ya que, puesto que Dios los creó, debe de haber tenido una razón suficiente para crear al primero de ellos y esta razón quedó satisfecha cuando lo creó, por lo que para crear al otro tuvo que tener una razón diferente, por más y que a nosotros esta razón se nos pueda escapar, dada la semejanza entre sustancias que, cabe agregar, es esencial a nuestro conocer. En el Escrito V a Clarke dice Leibniz:

Entre otras consecuencias, infiero de este principio (el de razón suficiente) que no hay en la naturaleza dos seres absolutos, reales, indiscernibles el uno del otro; porque si los hubiera, Dios y la Naturaleza actuarían sin razón al ordenar el uno en lugar del otro; y que, por lo tanto, Dios no produce dos porciones de materia perfectamente iguales y semejantes. ${ }^{13}$

A propósito de esta relación entre los dos principios, Leibniz le aclara a Clarke tres cosas que podrían suscitar reservas con respecto a su aceptación. La primera tiene que ver con el atomismo: los átomos podrían considerarse no sólo el fundamento último de la materia, sino un buen ejemplo de indiscernibilidad. De estas dos suposiciones se podría derivar la muy peregrina idea, según Leibniz, de que Dios no pudo haber creado la materia, puesto que se compone de átomos que son indiscernibles y que, por serlo, resultan inexistentes si se acepta el principio leibniziano. Para expresar esta idea, Leibniz cita las palabras de Clarke: "El argumento, si fuera bueno, probaría que es imposible que Dios crease la materia, ya que las partes perfectamente sólidas de la materia, si las consideramos de la misma figura y dimensión (lo que siempre es posible suponer) serían exactamente semejantes." ${ }^{14}$

Este texto nos conduce directamente a la segunda aclaración leibniziana, ya que es el mismo Clarke quien enfatiza que podemos suponer o concebir dos partes de materia perfectamente iguales: dos átomos, podríamos decir. La aclaración de Leibniz consiste en señalar que es obvio que podemos concebir in abstracto dos cosas perfectamente iguales, pero esto no

\footnotetext{
12 Aunque el PRS se suele asociar con el nombre de Leibniz, se puede encontrar en la filosofía medieval y se atribuye normalmente a Abelardo, quien, queremos suponer, lo debe haber formulado antes de que Fulber, tío de Eloísa, encontrara una razón suficiente para mandarlo castrar.

${ }^{13}$ Leibniz, Escrito V, 21, en Alexander (comp.), op. cit., p. 61.

${ }^{14}$ Ibid.
} 
quiere decir que existan. Por otro lado, el que tales pares —o tríos o infinitudes, ya que se está pensando en átomos- no existan no es casual, pues su existencia iría en contra del orden natural de las cosas y de la racionalidad de Dios, que no crea sin tener una razón. Dice Leibniz: "La suposición de dos indiscernibles tales como dos porciones de materia perfectamente semejantes parece claramente posible en términos abstractos; pero no es consistente con el orden de las cosas ni con la sabiduría divina, por la cual nada se admite sin razón." 15 De esta manera Leibniz puede establecer no sólo una relación entre el PII y el PRS, sino una relación entre un principio que no es ni empírico ni claramente lógico y la experiencia: "Cuando yo niego que existan dos gotas de agua perfectamente iguales o cualquier otro par de cuerpos indiscernibles el uno del otro, no estoy diciendo: es absolutamente imposible suponerlos, sino que esto es algo contrario a la sabiduría divina y, consecuentemente, no existe."16

La tercera aclaración de Leibniz se relaciona con una observación de Clarke acerca no tanto de la sabiduría divina como de su voluntad y su poder. La idea de Clarke sugiere preguntas como: ¿puede el PII ponerle un límite a la creación divina?; ¿está fuera del alcance de Dios crear dos cosas absolutamente iguales?, y, suponiendo que así fuera, ¿es esta imposibilidad del orden del deseo o del poder?; ¿no lo puede desear o no lo puede hacer? El tono de estos planteamientos refleja el paso de una argumentación en términos de teoría física y átomos a una argumentación en términos de las limitaciones de Dios. La idea de Clarke es que podemos aceptar una versión causal del principio de razón suficiente, pero que debemos subrayar que es una manifestación de la voluntad de Dios y no una restricción o condición que limitara su deseo o la muy divina capacidad de elección; aceptar la versión leibniziana del PRS es, para Clarke, tanto como abrirle las puertas a la pagana Fatalidad que, como se sabe, con el apoyo de Hera podía ponerle un "hasta aquí" al mismísimo y caprichoso Zeus. ${ }^{17}$ Dice Clarke:

Ciertamente es verdad que nada es sin una razón suficiente para que así sea y por la cual es así y no de otra manera. Y, por lo tanto, donde no hay causa no puede haber efecto. Pero esta razón suficiente no es, frecuentemente, otra cosa que la mera voluntad de Dios. Por ejemplo: ¿por qué este sistema particular de materia debería ser creado en un lugar particular y aquel en otro, cuando hubiera sido exactamente la misma cosa viceversa (ya que todo lugar es absolutamente indiferente para toda materia), suponiendo que los dos sistemas o particulares fueran semejantes?; no podría haber otra razón que la mera voluntad de Dios. El que ésta no pudiera actuar en ningún caso sin una causa predeterminada, como una balanza que no se puede mover sin un peso

15 Ibid.

${ }^{16}$ Leibniz, Escrito V, 25, en Alexander (comp.), op. cit., p. 62

${ }^{17}$ Véase, por ejemplo, Iliada, libro 16, 426-461. 
preponderante, esto tendería a quitarle todo el poder de elección y a introducir la fatalidad. ${ }^{18}$

De esta manera condicionada acepta entonces Clarke el PRS, lo cual era de esperarse, dada, como ya dijimos, la relación de este principio con la posibilidad y la plausibilidad de dar explicaciones. Lo curioso, pero no raro en la discusión de las ideas de Leibniz, es que la búsqueda de alternativas puede acercarnos a las ideas que intentábamos rechazar. En el caso de Clarke, su misma invocación de las posibilidades divinas lo acerca a aceptar la verdad empírica del PII, ya que, en rigor y en su teoría física, los átomos son postulados teóricos. Dice Clarke:

Ciertamente es verdad que ningún par de hojas y quizá ningún par de gotas son exactamente iguales, ya que son cuerpos muy compuestos. Pero el caso es muy diferente en las partes de la materia sólida simple. Y aun en los compuestos no es imposible que Dios haga dos gotas de agua exactamente iguales. E incluso si las hiciera exactamente iguales no por ello llegarían nunca a ser una y la misma gota de agua. ${ }^{19}$

Aquí, obviamente, no podemos sino hacer referencia a estos problemas de la voluntad y la omnipotencia divinas, pero aun así tenemos que recordar que se hacen con el telón de fondo de una distinción entre necesidad lógica y necesidad moral que, como la moral misma, ha perdido popularidad, pero que para Leibniz y su lectura es fundamental. Arguye Leibniz:

Hay necesidades que deben ser admitidas, porque hay que distinguir entre una necesidad absoluta y una necesidad hipotética. Hay que distinguir también entre una necesidad que tiene lugar porque su opuesto implica una contradicción (y a la que se llama lógica, metafísica o matemática) y una necesidad que es moral y que hace que el sabio escoja lo mejor y todo espíritu siga la inclinación más fuerte. ${ }^{20}$

A partir de esta distinción, Leibniz intentará mostrar que ni el PRS ni el PII ponen en tela de juicio la omnipotencia divina. La noción de necesidad moral es fundamental porque, en relación con la Divinidad, encadena su omnipotencia con su racionalidad y su voluntad con su bondad; por otro lado, en relación con sus creaturas, permite conciliar su conocimiento del destino de esas creaturas con la libertad que les es otorgada y que ya estaba considerada en su concepción. ${ }^{21}$ De esta manera, argüirá Leibniz, es un error pensar que la racionalidad que impone el PRS a la Divinidad es una

${ }^{18}$ Clarke, Réplica II, en Alexander (comp.), op. cit., pp. 20-21.

${ }^{19}$ Clarke, Réplica IV, 3 y 4, en Alexander (comp.), op. cit., p. 46.

${ }^{20}$ Leibniz, Escrito V, 4, en Alexander (comp.), op. cit., p. 56.

${ }^{21}$ Véase Leibniz, Escrito V, 6, en Alexander (comp.), op. cit., p. 56. 
limitación de su libertad, ya que la necesidad moral, que la lleva a actuar de la mejor manera, lejos de limitarla, la perfecciona, pues gracias a ella no puede evitar (empecher) actuar de la mejor manera. Dice Leibniz: "En cuanto a la necesidad moral, ésta no le quita nada a la libertad, porque cuando el sabio y, sobre todo, Dios (el Sabio soberano) escoge lo mejor, no es por ello menos libre; al contrario, el que no haya impedimento para actuar de la mejor manera es la libertad más perfecta." 22

Esta situación tiene su paralelo en la creaturas, que pueden imitar la sabiduría divina e inclinarse, en su humana medida, por el mayor bien; sin esta posibilidad no habría propiamente elección, ni humana ni divina, y reinaría el azar. De esta manera Leibniz pasa de la divina elección a la humana y la subsume también en la necesidad moral. Nos dice: "Y cuando cualquier otro elige según el bien más aparente y que con más fuerza le inclina, imita en ello la libertad del Sabio en la proporción de su disposición. Y sin ello la elección sería un azar ciego."23

Así, la elección, si lo es, no puede ser ciega, y menos aún si es divina y concierne a la creación; la clarividencia de Dios con respecto a sus creaturas es total en un sentido lo suficientemente fuerte del término como para dar qué pensar a un "holista" contemporáneo. En todo caso, queda claro que para Leibniz la necesidad moral está íntimamente ligada a la existencia de entidades contingentes, ya que éstas podrían no haber existido y su existencia depende de una elección divina y, subraya Leibniz, libérrima, puesto que ha sido perfeccionada por la necesidad moral. La existencia es, entonces, ese punto privilegiado donde se consolida la necesidad moral que da como resultado la creación y donde se inicia el dominio de la posibilidad y la necesidad lógicas; en este sentido, la necesidad moral es el horizonte que permite pensar en la razón suficiente de una entidad creada, ya que esta razón suficiente es, literalmente, su razón de ser. Precisamente por esto no debemos olvidar que la existencia tiene que ver con la necesidad moral y que, como enfatiza Leibniz, ésta es de un orden distinto del de la necesidad lógica, que tiene que ver con la esencia o con todo lo que tenga que ver con la caracterización de una entidad después de haber sido creada. Defendiéndose de las críticas de Clarke, dice Leibniz:

Pero decir que Dios no puede sino escoger lo mejor y de allí inferir que aquello que no escoge es imposible, es confundir los términos, el poder y la voluntad, la necesidad metafísica y la necesidad moral, las esencias y las existencias. Ya que lo que es necesario lo es por su esencia, puesto que su opuesto implica una contradicción; pero lo contingente que existe le debe su existencia al principio de lo que es mejor, razón suficiente de las cosas. ${ }^{24}$

${ }^{22}$ Leibniz, Escrito V, 7, en Alexander (comp.), op. cit., p. 56.

${ }^{23}$ Ibid., en Alexander (comp.), op. cit., pp. 56-57.

${ }^{24}$ Leibniz, Escrito V, 9, en Alexander (comp.), op. cit., p. 57. 
De esta manera se hace aparente la naturaleza fundante, y fundamental, del PRS: conformado según la necesidad moral, es un "principe des existences" o, más explícitamente en inglés, un "ground of existences": el fundamento de las existencias. ${ }^{25}$ También se clarifica el punto básico de la divergencia entre Clarke y Leibniz. Aquél, como ya vimos, había observado que, desde el punto de vista atomista, Dios no podría haber creado la materia, puesto que está compuesta de átomos, y estos átomos, argüía Clarke, podían concebirse exactamente iguales. ${ }^{26} \mathrm{Y}$ era esta posibilidad de concebir, in abstracto, la que cuestionaba la naturaleza lógica del principio de la identidad de los indiscernibles y la que obligaba a Leibniz a considerarlo como una consecuencia de un principio más fundamental, el de razón suficiente. Para Leibniz resulta claro que el carácter más fundamental del PRS no lo convierte en un principio lógico, pero sí permite introducir la ya mencionada distinción entre necesidad absoluta (metafísica o matemática) y necesidad hipotética o moral, y mencionar como paradigma de la primera el principio de identidad o contradicción y de la segunda el principio de razón suficiente: "la necesidad absoluta y metafísica depende de otro gran principio de nuestros razonamientos, que es el de las esencias, esto es, el de la identidad o contradicción: ya que lo que es absolutamente necesario es el único camino posible y su contrario implica una contradicción". ${ }^{27}$

Así, Leibniz puede aceptar, como Clarke, que el PRS y su derivado, el PII, no están sujetos a la necesidad absoluta o lógica; pero esto no los hace menos básicos y necesarios, ya que lo son desde el punto de vista moral que corresponde a la bondad de Dios. Dado este planteamiento, alguien podría pensar que la balanza, que tan importante le parecía a Clarke, se podría inclinar hacia éste, dada la considerable pérdida de peso de la bondad. Sin embargo, no podemos olvidar el peso ontológico y la prioridad epistemológica del PRS; como ya vimos, para Leibniz el PRS es el principio que fundamenta la existencia de los entes contingentes, esto es, no necesarios desde el punto de vista lógico. Lógica y existencia, ya se sabe, son nociones que tienden a suscitar tensiones; pero la lección leibniziana nos invita a prevenir que nuestros afanes lógicos nos lleven a ignorar o desplazar la existencia, que nos lleven a pensar que podemos hablar de mundos posibles sin establecer uno de ellos como punto de referencia previo. Este mundo, se puede argüir, es el que existe y es condición de posibilidad de la postulación de otros mundos - lógicamente posibles- que, se puede argüir también, no pueden ser sino variantes (lógicas) del mundo existente, y no postulado, del que partimos. ${ }^{28}$

${ }^{25}$ Véase Leibniz, Escrito V, 10, en Alexander (comp.), op. cit., p. 57.

${ }^{26}$ Véase Clarke, Réplica IV, 3 y 4, en Alexander (comp.), op. cit., p. 45.

${ }^{27}$ Leibniz, Escrito V, 10, en Alexander (comp.), op. cit., p. 57.

${ }^{28}$ Recientemente, las investigaciones de Kripke, y de la semántica de la lógica modal en general, han traído a cuento el problema de la naturaleza de los mundos posibles, ya que, por 
Por otro lado, que se suele asociar con la epistemología, tal vez se podría pensar en el PRS como la expresión de la idea de que hay razones y causas, y, por tanto, podría considerárselo como una forma de la hipótesis de racionalidad que, más allá de la existencia de un Dios racional y bondadoso, justifica nuestros afanes de dar una explicación del mundo en que vivimos y de las cosas, cercanas o lejanas, que nos rodean. El principio, leibnizianamente formulado, se suele asociar con otras tesis de Leibniz y frecuentemente se le juzga con los cándidos ojos con que lo vio Voltaire, ${ }^{29}$ pero no es ésta la única forma de pensarlo ni de pensar el espíritu de la filosofía leibniziana. Recuérdese, por ejemplo, y en relación con el juicio de Voltaire, que si bien podemos pensar que afirmar que éste es el mejor de los mundos posibles es dar muestra de un optimismo que raya con la imbecilidad, también podemos pensar que es una muestra de un muy lúcido pesimismo. La diferencia puede ser tan simple como distinguir entre creer y saber: el optimista cree que éste es el mejor de los mundos posibles; el pesimista lo sabe.

El optimismo y el pesimismo, podríamos decir, son las dos caras que un ser humano puede poner ante el hecho del mundo, más allá o más acá de cualquier predicación, positiva o negativa. Las versiones religiosas, místicas, estéticas y existenciales de esta escena de conciencia del mundo son frecuentes; pero la que apunta Leibniz, me parece, está en relación con la existencia como presupuesto de la predicación y la posibilidad de diferencia. Es por esto que la existencia no cae en el ámbito de la necesidad lógica, ni en la reflexión de las esencias y la predicación propiamente dicha. Es por esto también que la descripción "el mejor de los mundos posibles" puede pensarse frente al horizonte de la posibilidad de concebir variantes distintas, y tal vez mejores, de este mundo; pero también puede pensarse que enfatiza que es el mundo que existe, el único que tenemos aunque no lo pensemos. Es el mejor porque existe y porque, mal que bien, e ignorando la sabiduría de Sileno, en él existimos y, gracias a ello, podemos pensarnos distintos y en otro lugar. Claro está que alguien nos podría reprochar no tanto nuestro optimismo o nuestro pesimismo, sino nuestro monismo moral, recordándonos que en este valle ni la miseria ni la fortuna nos tocan a todos por igual y que, en este sentido, los mundos del miserable y del afortunado pueden ser dos mundos aparte. Wittgenstein, por ejemplo, quien en su juventud tuvo sus momentos leibnizianos, ${ }^{30}$ hablaba en el Tractatus de estos mundos: el de los felices (Glückliche) y el de los infelices

ejemplo, hay filósofos que asumen una posición platónica y piensan que su importancia está en relación con la posibilidad de independizarlos del mundo existente, mientras que otros asumen una posición constructivista o formalista. Véase, S. Kripke, "Naming and Necessity", y D. Lewis, Conterfactuals, esp. el cap. 4.

${ }^{29}$ Véase Voltaire, Candide, cap. 1, y la entrada "Bien (tout est)" de su Dictionnaire Philosophique.

${ }^{30}$ Piénsese, por ejemplo, en sus ideas del análisis, los simples y el orden lógico. 
(Unglückliche). En ese libro afirma: "El mundo de los felices es distinto del mundo de los infelices." 31

Está claro, sin embargo, que esta distinción no afecta la unidad del mundo, sino que apunta a otro problema: el de la relación de los sujetos - creaturas, diría Leibniz - con el mundo, aunque parte del problema consiste en determinar qué clase de relación es la que se está considerando. Esta dificultad se hace patente cuando tratamos de explicar la diferencia entre el mundo de los felices y el de los infelices en términos de diferencias físicas, sociales, históricas o incluso psicológicas, ya que no parece que Wittgenstein estuviera pensando en alguna de ellas. ${ }^{32}$ Pero si no se trata de establecer la diferencia en los términos de estos conocimientos, entonces tal vez tengamos que pensar que la distancia que establece Wittgenstein entre la felicidad y la miseria pertenece a un ámbito distinto, en el sentido de que no es reducible a ningún saber teórico específico y en el sentido de que tiene que ver con la manera en que asumimos la existencia, nuestra y del mundo, más allá o antes de posibles afanes de cambio y transformación. Esta dicotomía entre analizar o pensar el mundo y asumir su existencia podría tal vez asimilarse, o al menos relacionarse, con algunas afirmaciones de Wittgenstein acerca del sentido del mundo y con lo que llegó a considerar su principal propuesta y problema cardinal de la filosofía: la distinción entre lo que se puede expresar y, por lo tanto, pensar, y lo que no se puede expresar sino sólo mostrar y, paralelamente, podríamos agregar, sólo asumir. ${ }^{33}$ En el Tractatus dice Wittgenstein:

6.41 El sentido del mundo debe quedar fuera del mundo. En el mundo todo es como es y sucede como sucede; en él no hay ningún valor, y aunque lo hubiese no tendría ningún valor.

Si hay un valor que tenga valor, debe quedar fuera de todo lo que ocurre y de todo ser-así.

Por otro lado, nada podría parecer más alejado de la posición de Wittgenstein que una distinción leibniziana entre necesidad lógica y necesidad moral. Cuando Wittgenstein - tratando tal vez de esclarecer la sombra de Schopenhauer - trae a cuento la voluntad, subraya - aun cuando es evidente que no se trata de la voluntad divina, sino de la humana- que ésta

${ }^{31}$ L. Wittgenstein, Tractatus Logico-Philosophicus, 6.43.

${ }^{32}$ Véase G.E.M. Anscombe, An Introduction to Wittgenstein's Tractatus, especialmente el cap. 13.

${ }^{33}$ En su libro sobre el Tractatus, Elizabeth Anscombe cita una carta de Wittgenstein a Russell donde le dice: "Me temo realmente que no has acabado de entender mi propuesta; todo el asunto de las proposiciones lógicas es sólo un corolario de ella. El punto principal es la teoría de lo que puede ser expresado (gesagt) por la proposiciones, esto es, por el lenguaje (lo que viene a ser lo mismo, lo que puede ser pensado) y lo que no puede ser expresado por las proposiciones, sino sólo mostrado (gezeigt); esto, creo, es el problema cardinal de la filosofía" (Anscombe, op. cit., p. 161). 
no puede cambiar el mundo. El énfasis no es retórico; está relacionado con la naturaleza del mundo y de la voluntad: mundo y voluntad son de tal manera independientes, que aun cuando, por una especie de milagro, se estableciera una perfecta armonía entre nuestro deseo y la realidad, esta coincidencia sería precisamente eso, una coincidencia, un accidente, una merced de la suerte (eine Gnade des Schicksals), "una gracia del destino", como traduce Elizabeth Anscombe. ${ }^{34}$ Dice Wittgenstein en el Tractatus:

6.373 El mundo es independiente de mi voluntad.

6.374 Aunque todo lo que deseáramos ocurriese, esto sería solamente, por así decirlo, una merced de la suerte, pues no hay conexión lógica entre voluntad y mundo que pueda garantizar tal cosa, ni nosotros podríamos a su vez querer esta supuesta conexión física.

Esta separación radical entre mundo y voluntad, debemos recordarlo, no hace referencia a una noción psicológica, sino ética de la voluntad y, en consecuencia, desde el punto de vista wittgensteniano, la pone en el ámbito de lo que no se puede decir:

6.423 De la voluntad como sujeto (Träger) de la ética (des Ethischen) no se puede hablar.

Y la voluntad como fenómeno sólo interesa a la psicología.

De esta manera, aun cuando no podemos establecer un paralelo que relacione cabalmente la distinción de Leibniz entre necesidad lógica y necesidad moral, con la de Wittgenstein entre mundo y voluntad moral, sí podemos notar que ambos tratan de establecer dos órdenes o ámbitos: el mundano, sujeto a las leyes físicas y lógicas, y el moral, sujeto, según Leibniz, al principio de razón suficiente y situado, para Wittgenstein, fuera del mundo, fuera del ámbito de lo que se puede decir, pero que, no obstante, está relacionado con la posibilidad de darle un sentido al mundo y de caracterizar la ética como transcendental. Dice Wittgenstein:

6.41 Pues todo lo que ocurre y todo ser-así es casual (zufällig).

Lo que lo hace no casual no puede quedar (liegen) en el mundo, pues de otro modo sería a su vez casual.

Debe quedar fuera del mundo.

6.42 Por lo tanto, tampoco puede haber proposiciones de ética. Las proposiciones no pueden expresar nada más alto.

6.421 Es claro que la ética no se puede expresar.

La ética es transcendental.

La posibilidad de una correspondencia entre las distinciones leibniziana y wittgensteiniana del orden lógico y el orden moral no debe hacernos olvidar que la concepción de Leibniz de la lógica concierne a lo que él llama

${ }^{34}$ Anscombe, op. cit., p. 169

Diánoia, vol. XLIX, no. 52 (mayo 2004). 
las esencias, mientras que la de Wittgenstein se estructura alrededor de la noción de tautología. ${ }^{35}$ Sin embargo, ambos coinciden en suponer que aunque la lógica pueda concebirse independiente de la experiencia, tiene que partir de algo dado, de algo, pensaría Leibniz, creado. Dice Wittgenstein:

5.552 La 'experiencia', de la que tenemos necesidad para entender la lógica, no es que algo ocurra de tal y tal modo, sino que algo es; pero esto no es experiencia.

La lógica precede a toda experiencia: que algo es así.

Es antes que el Cómo, no que el Qué.

Queda claro, entonces, que el lugar de la lógica no se encuentra, para Wittgenstein, en el ámbito de la experiencia, sino que la precede; pero hay que notar que esta experiencia la caracteriza Wittgenstein como que las cosas o el mundo sean de una determinada manera: "que algo es así". O sea que esta experiencia es distinta de aquella "de la que tenemos necesidad para entender la lógica", pues esta "experiencia" —entre comillas— no tiene que ver con el cómo sean las cosas en el mundo, con "que algo es así", sino con que algo sea, o mejor dicho, con "que algo es", sin subjuntivos que opaquen la pureza del existencial.

Así, si la experiencia de lo que es es necesaria y precede al cómo, y si lo místico está relacionado con la existencia no cualificada del mundo, entonces tal vez no sea del todo aventurado sugerir un paralelo de la distinción de Wittgenstein entre lo que se puede decir y lo místico (de lo que no se puede hablar) con la distinción de Leibniz entre lo lógico y lo moral. Ambos, podríamos decir, coinciden no sólo en la importancia de la reflexión lógica y científica acerca de las esencias y del cómo en general, sino en la importancia de la existencia no cualificada del mundo como punto de referencia para hablar tanto de mundos posibles como del mundo que nos permite calificarlos de posibles. Es en este sentido que la lógica precede al Cómo, pero no al Qué; está en los límites de la experiencia, ya que su supuesto fundamental y fundante es, como en Leibniz, que algo es. Este "es" precede a la experiencia, en el sentido de cómo es el mundo, y a la lógica en el sentido de que su "experiencia" es necesaria para entender la lógica, por más que esa experiencia no pueda serlo en el sentido cabal del término; en el sentido cabal entramos en el ámbito de lo místico y de su "experiencia" como condición de comprensión. Enfatiza Wittgenstein:

\subsection{No es lo místico cómo sea el mundo, sino que sea.}

(La dificultad de aceptar esta posición se refleja en la traducción castellana, que con un solo acento sobre el "que" convierte el misticismo en un afán científico.)

35 Véase Tractatus, 6.1-6.13. 
Por su propia naturaleza, este tipo de planteamientos hace dudosa casi cualquier comparación que trate de establecer algún paralelo entre el orden moral leibniziano y el orden místico wittgensteiniano. Sin embargo, como ya señalamos, parece claro que ambos apuntan al reconocimiento de una prioridad ontológica y moral de la existencia, en su sentido más lato, sobre la lógica y el análisis de las esencias, como diría Leibniz, o del cómo, como diría Wittgenstein. A qué orden pertenezca esta coincidencia tampoco es fácil de determinar, pero sí es de notarse que no podemos extenderla directamente a una convalidación del PII; no lo podemos hacer porque, en su punto esencial, Wittgenstein usó el mismo argumento que Clarke para rechazar el principio y, con él, la definición de la identidad de Principia.

Como ya dijimos al inicio de este trabajo, la definición de identidad formulada en Principia se puede descomponer en dos implicaciones, una de las cuales, la que afirma que si $x$ y y tienen todas sus propiedades en común, entonces son iguales, se puede identificar con el PII. Más adelante vimos también que, entre otras cosas, Clarke objetó a Leibniz que bien se podría concebir que dos cosas tuvieran todas sus propiedades en común sin que este acto de pensamiento pudiera considerarse como una contradicción; esta posibilidad, de acuerdo con los propios lineamientos leibnizianos acerca de la naturaleza de la necesidad lógica, indica que el principio no es un principio lógico. Paralelamente, Wittgenstein encuentra inadecuada la definición de Russell porque, de acuerdo con ella, no podríamos afirmar que dos objetos difieran solo numero y señala, también como Clarke, que lo determinante no es la verdad o la falsedad de la afirmación, sino que tenga sentido el hecho, de acuerdo con Clarke, de poder pensarla sin contradicción. Por su parte, dice Wittgenstein:

5.5302 La definición de Russell de '=' no basta (genügt nicht); porque, según ella, no se puede decir que dos objetos tengan todas sus propiedades en común. (Incluso si esta proposición no es nunca verdadera (richtig), tiene, sin embargo, sentido.)

Esto es, la comunidad de propiedades no puede implicar necesariamente la identidad, porque la posibilidad de su excepción, que dos objetos tuvieran todas sus propiedades en común, está garantizada por su plausibilidad. Para Clarke, ya vimos, esta plausibilidad se refleja en la posibilidad de concebirla; para Wittgenstein, en la posibilidad de afirmarla con sentido. Y ambas posibilidades, podríamos agregar, descansan en el principio leibniziano de que la contingencia de una proposición se muestra en el hecho de que ni ella ni su negación sean contradictorias. Para Wittgenstein las contradicciones, como las tautologías, aunque estrictamente no se pueden considerar sinsentidos, sí carecen de él. Dice Wittgenstein:

4.461 La proposición muestra aquello que dice; la tautología y la contradicción muestran que no dicen nada. 
La tautología no tiene condiciones de verdad, pues es incondicionalmente verdadera; y la contradicción, bajo ninguna condición es verdadera.

La tautología y la contradicción carecen de sentido (sinnloss).

4.4611 Tautología y contradicción no son, sin embargo, sinsentidos (unsinnig); pertenecen al simbolismo, del mismo modo que cero ("0") es parte del simbolismo de la aritmética.

En consecuencia, el PII, que tiene sentido y no es una tautología, no puede ser una afirmación lógica, sino una contingencia; sin embargo, no es una contingencia normal sino anómala, en la medida misma de los señalamientos, tanto de Clarke ${ }^{36}$ como de Wittgenstein, con respecto a su verdad o, si se quiere, con respecto a la improbabilidad de su falsedad. Si el PII fuera una tautología no tendría, para Wittgenstein, condiciones de verdad, sino que sería incondicionalmente verdadera; pero entonces, ya vimos, carecería de sentido. Wittgenstein sostiene que su negación, la proposición que afirma que dos objetos tienen todas sus propiedades en común, tiene sentido no obstante que tal vez nunca sea verdadera, con lo cual, podríamos pensar, se acerca a una especie de contradicción, aunque, obviamente, no de raigambre lógica. Confinada entre paréntesis y con la cotidianidad de la palabra "richtig" (que Tierno Galván, muy filosóficamente tradujo como "verdadera"), se encuentra la acotación wittgensteiniana: "(Incluso si esta proposición no es nunca verdadera (richtig), tiene, sin embargo, sentido (Sinn))."37

¿Cuál es, entonces, el estatus del PII o de su negación? Decir que se trata de una contingencia invita, ya vimos, a hacer precisiones de la noción de tautología que no se pueden conciliar fácilmente ni son fáciles de establecer a partir de la noción wittgensteiniana de proposición. Parte de la dificultad de aclarar la naturaleza del PII tiene que ver, dentro del contexto del Tractatus, con que la excepción a este principio se introduce como la posibilidad de decir, o afirmar, la diferencia solo numero. Esta diferencia, aunque peculiar, tiene sentido y la podemos concebir consecuentemente para Wittgenstein y básicamente para Clarke. La relación entre la posibilidad, el sentido y la proposición es, para Wittgenstein, que el sentido de la proposición está relacionado con la posibilidad de la situación. Dice Wittgenstein:

2.221 Lo que la figura (Bild) representa es su sentido.

3.001 'Un hecho atómico (Sachverhalt; estado de cosas) es pensable' significa: Nosotros podemos figurárnoslo.

${ }^{36}$ Véase, por ejemplo, Clarke, Réplica IV, 3 y 4, en Alexander (comp.), op. cit., pp. 45-46.

${ }^{37}$ Tractatus, 5.5302. 
3.11 Nosotros usamos el signo sensiblemente perceptible de la proposición (sonidos o signos escritos, etcétera) como una proyección del estado de cosas posible (als Projektion der mögliche Sachlage).

Podríamos decir, entonces, que la posibilidad de que dos cosas tengan todas sus propiedades en común sea un estado de cosas concebible se puede expresar, en términos wittgensteinianos, diciendo que nos podemos "figurar" tal situación y que esta figuración se proyecta en la proposición que afirma la posibilidad de tal estado de cosas. Claro está que la imagen figurada no necesariamente tiene que ser "retrato de una cosa", como diría fray Luis de León, y esta posibilidad es esencial para distinguir entre proposiciones verdaderas, retratos fieles, podríamos decir, y proposiciones falsas, más fieles a la fantasía que a la realidad. Así, desde este punto de vista, por un lado podríamos pensar que el PII es una proposición donde se proyecta la posibilidad de un estado de cosas y que, como tal, bien podría ser el caso que ese estado de cosas no se diera; esto es, que existieran dos cosas distinguibles solo numero. Lo curioso de esta contingencia, ya señalamos, es que tanto Clarke como Wittgenstein la critican por no ser una verdad lógica, pero sin dejar de mencionar que probablemente es verdadera. Podemos entonces aceptar con Clarke y Wittgenstein que el PII no es un principio lógico, y menos una tautología, pero también pensar, sin contradecirlos, que es una verdad. El problema obviamente sigue siendo qué clase de verdad es el PII; pero cabría pensar que se trata de un problema con un trasfondo wittgensteiniano. Un primer intento en esta dirección es tomar en cuenta que el estado de cosas cuya posibilidad se proyecta y se prohíbe en el PII es la existencia de objetos completamente iguales o, como hemos venido diciendo, que difieran solo numero. En relación con estos aspectos del problema, la posición de Wittgenstein es bastante explícita: no piensa que se pueda establecer una relación de identidad entre objetos ni que se pueda hablar de una relación de identidad con un objeto determinado:

5.5301 Que la identidad no es una relación entre objetos es obvio. Esto se esclarece, por ejemplo, completamente si consideramos la proposición “( $x): f x$. $\supset . x=a$ ". Lo que esta proposición dice es simplemente que sólo a satisface la función $f$, y no que sólo aquellas cosas que tienen una cierta relación con $a$ satisfagan la función $f$.

Se podría naturalmente decir que en efecto solamente a tiene esta relación respecto de $a$, pero para expresar esto necesitaríamos del propio signo de identidad. ${ }^{38}$

Esta línea de pensamiento lo lleva a considerar la negación propiamente leibniziana del PII, esto es, la afirmación de la existencia de dos cosas completamente iguales y a considerarla como un sinsentido; este sinsentido apunta hacia la sorpresa humeana con respecto a la autoidentidad.

${ }^{38}$ Tractatus, 5.5301. 
(La otra forma de cuestionar el PII, debemos recordar, es la de Clarke, que afirma que podemos concebir objetos que difieran solo numero y que, como ya vimos, el mismo Wittgenstein usa para criticar la definición de Russell.) Con respecto a la existencia de pares idénticos, dice Wittgenstein:

5.5303 Sea dicho de paso: decir de dos cosas que son idénticas es un sinsentido, y decir de una que es idéntica consigo misma no es decir nada.

Pero, si afirmar la identidad de dos cosas es un sinsentido, ¿cómo podremos calificar desde este punto de vista el PII, que es la afirmación acerca de que no existe en la naturaleza tal par de cosas? ¿Por qué, podríamos insistir, es un sinsentido afirmar que dos cosas son idénticas? Puesto que Wittgenstein enfatiza el "dos", se podría pensar que está siguiendo la intuición básica leibniziana: si son dos, deben ser distintas. Claro está que, para Leibniz, el "deben" se deriva de la naturaleza bondadosa de Dios y es, por lo tanto, un principio moral, mientras que la razón que pudiera tener Wittgenstein para asumir la misma intuición no es tan transparente. O tal vez, podríamos elucubrar, no se trata precisamente de una razón, en el sentido de una explicación derivada de una reflexión newtoniana acerca de las propiedades de las cosas, sino de una limitación relacionada con la noción misma de "cosa" que podría emparentarse con otros problemas del Tractaus, como los de la forma lógica, lo inexpresable y, especialmente, el del ámbito que Wittgenstein calificaba de místico. Lo místico, recordemos, puede presentarse de varias maneras; aunque no es expresable, tiene que ver con la posibilidad de que las proposiciones - contingentes- tengan sentido, vía la forma lógica, y con que el mundo - contingente y casual que es- pueda tenerlo. Un punto inicial de esta argumentación es la noción de imagen (Bild) —en el sentido amplio de representación-, que le permite a Wittgenstein introducir una noción bastante radical de forma lógica; la forma lógica se presenta como la condición de posibilidad de la representación y, en la misma línea, se la identifica con la forma de la realidad:

2.18 Lo que cada figura (Bild), de cualquier forma, debe tener en común con la realidad para poderla figurar por completo - justa o falsamente- es la forma lógica, esto es, la forma de la realidad.

Hay que notar, sin embargo, que lo que representa una figura es literalmente una posibilidad, una de la formas que podrían tomar los hechos, no su existencia.

2.201 La figura, figura la realidad representando una posibilidad de la existencia y de la no existencia de los hechos atómicos.

La existencia o no existencia de un hecho atómico, acorde con una representación, es el fundamento de nuestro hablar de verdades y falsedades: 
2.221 Lo que la figura representa es su sentido.

2.222 En el acuerdo o desacuerdo de su sentido con la realidad, consiste su verdad o falsedad.

Bajo estos lineamientos, como se sabe, Wittgenstein transformará lo que podría parecer una manifestación más de la teoría de la adecuación en una teoría de las proposiciones como figuras de la realidad que no sólo pondrá el acento en aquello que hace posible la representación —la forma lógica一, sino que, giro notable, pondrá esta condición fuera de los límites de la representación. Y puesto que las proposiciones pueden representar toda la realidad, la forma lógica no podrá ser representada, a menos que tengamos acceso a un punto de vista externo, fuera de la realidad, de la lógica y del mundo.

4.12 La proposición puede representar toda la realidad, pero no puede representar lo que debe de tener de común con la realidad para poderla representar: la forma lógica.

Para poder representar la forma lógica debemos poder colocarnos con la proposición fuera de la lógica; es decir, fuera del mundo. ${ }^{39}$

Pero si la forma lógica queda fuera del mundo queda entonces fuera del ámbito de lo representable; aunque, al ser condición de la representación, se reflejará en la proposición y en el lenguaje en general. Este curioso estatus de la forma lógica, de ser condición de la representación pero ser ella misma irrepresentable, de estar esencialmente presente en el lenguaje sin nunca poder llegar a ser tema de conversación, le permite a Wittgenstein introducir la distinción entre el ámbito de lo que podemos hacer objeto de discurso y el de lo que sólo se expresa o se muestra en el lenguaje. Y lo que se muestra en el lenguaje, no debemos olvidar, es la forma lógica que, como ya vimos, Wittgenstein identifica con la de la realidad:

4.121 La proposición no puede representar la forma lógica; (la forma lógica) se refleja en ella (la proposición).

Lo que en el lenguaje se refleja, el lenguaje no puede representarlo.

Lo que en el lenguaje se expresa, nosotros no podemos expresarlo por el lenguaje.

La proposición muestra la forma lógica de la realidad.

La exhibe. ${ }^{40}$

Así las cosas, tal vez podríamos decir que, en un sentido, el PII está relacionado precisamente con la forma ontológica de la realidad y que Leibniz trató de expresar esta limitación ("no puede haber...") en un principio

\footnotetext{
39 Traducción modificada, pues Tierno Galván escribe: "pero no puede representar lo que debe tener de común con la realidad para poder representar la forma lógica", e ignora así la imposibilidad, expresada en el siguiente párrafo, de colocarnos fuera del mundo.

${ }^{40}$ Tractatus, 4.121.
} 
que, siguiendo los criterios del Tractatus, debería rayar con lo inexpresable. Leibniz vio también que el PII no se podía expresar como una verdad lógica, pero destacó el ámbito de su necesidad al señalar que era un principio que, no obstante no alcanzar la necesidad lógica, sí participaba de la necesidad moral. Y, de acuerdo con la filosofía leibniziana, esta necesidad moral tiene precedencia sobre la necesidad lógica. Dicho de otra manera: Leibniz afirma, en un principio de carácter ontológico-moral, lo que Wittgenstein cree que no se puede expresar por medio del lenguaje sino sólo mostrar, por más que lo que se muestre se pudiera identificar con el contenido del principio leibniziano. Esta posible identificación está relacionada con lo que Wittgenstein cree necesario mostrar en relación con la identidad y que lo lleva a formular un simbolismo, alternativo al russelliano, bajo los lineamientos del PII leibniziano. Esto es, en lugar de formular en términos lógicos una caracterización de la identidad, Wittgenstein hará que su simbolismo la muestre. Dice Wittgenstein:

5.53 Expreso la identidad del objeto por la identidad del signo y no por medio de un signo de identidad. $Y$ la diversidad de los objetos por la diversidad de los signos.

5.531 Yo no escribo, pues, " $f(a, b) . a=b$ ", sino " $f(a, a)$ " (o " $f(b, b)$ "). Y no " $f(a, b) . \sim a=b "$, sino " $f(a, b)$ ".

5.532 Y análogamente: no " $(\exists x, y) \cdot f(x, y) \cdot x=y$ ", sino " $(\exists x) \cdot f(x, x)$ "; y no " $(\exists x, y) . f(x, y) . \sim x=y$ ", sino " $(\exists x, y) . f(x, y)$ ".

(Y así, en lugar del russelliano " $(\exists x, y) . f(x, y)$ ”; “ $(\exists x, y) . f(x, y) \cdot \vee$ . $(\exists x) . f(x, x) ")$

La cercanía de estos planteamientos al PII es notoria, aun cuando no se establezca desde el punto de vista ontológico leibniziano, ya que para Wittgenstein la identidad no es una relación entre objetos. Esta conclusión, que a Wittgenstein le parece obvia, se menciona, como ya vimos, un poco antes de hacer referencia, en passant, al "sin sentido" que sería afirmar un contraejemplo del PII y a la pobreza de contenido de una afirmación de autoidentidad. ${ }^{41}$

Claro está que se podría argüir que, desde un punto de vista estrictamente wittgensteiniano, lo que concierne a lo místico no puede ser afirmado, sino sólo mostrado, y que, por tanto, cualquier intento de acercar una tesis explícita, como el PII, a ese ámbito es violentar la posición wittgensteiniana. Sin embargo, y como se sabe, esta violencia la inició el propio Wittgenstein en el Tractatus al tirar su famosa escalera y sugerirnos una contemplación silenciosa de un mundo que esa escalera nos había permitido vislumbrar:

${ }^{41}$ Véase Tractatus, 5.5301, 5.5303.

Diánoia, vol. XLIX, no. 52 (mayo 2004). 
6.54 Mis proposiciones son esclarecedoras de este modo; que quien me comprende acaba por reconocer que carecen de sentido, siempre que el que comprenda haya salido a través de ellas fuera de ellas. (Debe, pues, por así decirlo, tirar la escalera después de haber subido.)

Debe superar estas proposiciones; entonces tiene la justa visión del mundo.

Leibniz, podríamos pensar, también nos permite atisbar ese mundo, pero sin invitarnos a hacer un voto de silencio. Muy por el contrario, en ese umbral, anterior a la reflexión de las esencias, es donde se encuentra para él, y tal vez también para Wittgenstein, el fundamento y la fundamentación de una filosofía que no se confunda con la reflexión científica. El mundo es y es distinto de todo otro mundo concebible en la precisa medida, arguye Leibniz, de que existe. Se pueden concebir otros mundos, muy distintos, muy parecidos e incluso iguales a éste, pero sólo éste existe. Y ésta es, para Leibniz, una verdad importante y expresable. Wittgenstein, por otro lado, acepta que el mundo es y afirma que es todo, "lo que acaece" (was der Fall ist), y lo identifica con "la totalidad de los hechos", ${ }^{42}$ aunque esta totalidad, o el hecho de esta totalidad, no sea propiamente un hecho. Las resonancias russellianas de este hablar de totalidades cambian de tono cuando notamos que, para Wittgenstein, la totalidad limitada del mundo, más que generar paradojas, propicia la experiencia de lo místico. Esta experiencia se relaciona tanto con el mundo como con la vida, ya que en este nivel mundo y vida se identifican:

$6.45[\ldots]$ Sentir el mundo como un todo limitado es lo místico.

$5.621 \mathrm{El}$ mundo y la vida son una sola cosa. (Die Welt und das Leben sind Eins.)

Lo místico, ya vimos, no es "cómo sea el mundo, sino que sea" y, por consiguiente, no puede ser uno de los hechos del mundo ni expresarse como tal; sólo puede mostrarse:

6.522 Hay, ciertamente, lo inexpresable (Unaussprechlichen), lo que se muestra (zeigt) a sí mismo; esto es lo místico.

Por otro lado, como ya señalamos, para Wittgenstein el sentido del mundo, si es que lo tiene, no puede encontrarse en el mundo ni en cómo sea el mundo, pues éste es el ámbito de la contingencia; debe quedar entonces "fuera del mundo", y es por ello inexpresable; pero también es referencia de valor y sentido que podríamos identificar con lo místico, con la existencia no cualificada del mundo, aquella que para Leibniz cae dentro del orden de la creación y la necesidad moral. Para Leibniz, la existencia también precede al análisis, pero no sólo en el estricto sentido ontológico de que el mundo existe, sino en el sentido de que hay una razón para que exista, una razón

${ }^{42}$ Tractatus, 1, 1.1. 
para distinguirlo como único dentro de la gama de mundos posibles que este mundo nos permite concebir y pensar. Así, aceptar la existencia del mundo como vía mística de sentido del mundo vincularía tal aceptación con el principio de razón suficiente leibniziano, que se podría ver, precisamente, como la posibilidad misma de darle sentido al mundo en el que vivimos, por más contingente y casual que sea. El orden moral de Leibniz, el orden de la creación limitada por la bondad y la razón, se orientaría hacia el ámbito ético, hacia la posibilidad de vivir el mundo de los felices, de hacer coincidir el mundo limitado con las limitaciones de la vida, ya que "El mundo y la vida son uno".

Según los propios lineamientos leibnizianos y, obviamente, según los de Wittgenstein, ésta no es una buena razón para considerar como principio lógico el principio que afirma que todo tiene una razón; pero sí es una buena razón para simplemente considerarlo, para no descartarlo demasiado rápidamente en aras de una necesidad no sólo lógica, sino excluyente. Esta necesidad, se sabe, es la que prevalece en los contextos que, como Principia Mathematica, aspiran al máximo rigor lógico, aunque en algunos casos, como el de Principia mismo, se encuentren contaminados por principios que, como el de los indiscernibles o el de infinitud, difícilmente pueden aspirar a reducirse a una estructura tautológica. El que ésta sea la necesidad que prevalece es también la buena razón por la que la sensatez de Clarke haya prevalecido sobre la especulación de Leibniz y las críticas de Wittgenstein se hayan impuesto sobre los afanes de Russell, aunque, como hemos visto, el precio todavía no sea muy claro. Por otro lado, estas catedrales lógicas pueden ser un buen lugar para detenerse y reflexionar sobre las insistencias de Leibniz y Russell de redistribuir altares y reacomodar capillas. O tal vez, más simplemente, sean un buen lugar para reflexionar, con Wittgenstein, sobre la existencia del mundo y su catedral. En todo caso, aquí nos ha permitido atisbar el tamaño de la apuesta ontológica que encierra la definición russelliana de la identidad y apunta nuestra atención hacia la relación entre principios (morales) de existencia y principios lógicos (que suponen la existencia).

\section{BIBLIOGRAFÍA}

Alexander, H.G. (comp.), The Leibniz-Clarke Correspondence, Manchester University Press, Manchester, 1956.

Anscombe, G.E.M., An Introduction to Wittgenstein's Tractatus, Hutchinson, Londres, 1967.

Ishiguro, Hidé, Leibniz's Philosophy of Logic and Language, Duckworth, Londres, 1972.

Kripke, S., "Naming and Necessity", en D. Davidson y G. Harman (comps.), Semantics of Natural Language, Reidel, Dordrecht, 1972, pp. 253-355. [Versión en 
castellano: El nombrar y la necesidad, 2a. ed., trad. Margarita M. Valdés, Instituto de Investigaciones Filosóficas-UnAM, 1995.]

Leibniz, Discurso de metafísica, trad. Alfonso Castaño Piñán, Aguilar, Buenos Aires, 1962 (1685-1686).

—_, Monadología, trad. Manuel Fuentes Benot, Aguilar, Buenos Aires, 1964 (1714).

Lewis, D., Conterfactuals, Blackwell, Oxford, 1973.

Parkinson, G.H.R., Logic and Reality in Leibniz's Metaphysics, Oxford University Press, Oxford, 1965.

Russell, B., A Critical Exposition of the Philosophy of Leibniz, 2a. ed., George Allen and Unwin, Londres, 1937.

Voltaire, Candide, ou l'optimisme (1758), en CEuvres complètes de Voltaire/Complete Works of Voltaire, ed. Ulla Kölving et al., Voltaire Foundation, Ginebra/Banbury/ Oxford, 1968-, vol. 48, pp. 115-260.

_- Dictionnaire philosophique (1752), en OEuvres complètes de Voltaire/Complete Works of Voltaire, ed. Ulla Kölving et al., Voltaire Foundation, Ginebra/Banbury/ Oxford, 1968-, vols. 35-36.

Wittgenstein, Ludwig, Tractatus Logico-Philosophicus, trad. Enrique Tierno Galván, introd. Bertrand Russell, Revista de Occidente, Madrid, 1957.

Recibido el 17 de junio de 2003; aceptado el 2 de diciembre de 2003. 